\title{
Solid lipid nanoparticle induced apoptosis of macrophages via a mitochondrial-dependent pathway in vitro and in vivo
}

This article was published in the following Dove Press journal: International Journal of Nanomedicine

\author{
Wan-Li Liang ${ }^{l} * *$ \\ Lan Xiao ${ }^{2, *}$ \\ Hong-Wei Gu' \\ Xiao-Jun $\mathrm{Li}^{1}$ \\ Yu-Sang Li' \\ Wei Kevin Zhang' \\ He-Bin Tang' \\ 'School of Pharmaceutical Sciences, \\ South-Central University for \\ Nationalities, Wuhan 430074, People's \\ Republic of China; ${ }^{2}$ Department of \\ Traditional Chinese Medicine, Third \\ Xiangya Hospital, Central South \\ University, Changsha 410013, People's \\ Republic of China \\ *These authors contributed equally to \\ this work
}

Background and aims: Lipid nanoparticles (LNs) are widely applied in drug delivery systems because they can incorporate and stabilize lipophilic and hydrophilic molecules. LNs are generally considered quite safe and convenient for in vivo applications. However, we previously observed that certain types of LNs could cause a loss of Kupffer cells, a kind of resident macrophage in the liver. As a result, we investigated the details of this phenomenon. Methods: MTT assay, Annexin-V-FITC/PI double staining, JC-1 staining, flow cytometry, Western blot and transmission electron microscopy were used in cell-based experiments. Additionally, serum biochemical analyses, H\&E staining and immunofluorescence staining were performed to detect the acute and chronic changes of tissue structure and the number of Kupffer cells in mouse liver tissue samples.

Results: Application of LN depolarized and swelled the mitochondria of Raw264.7 cells, and disrupted the balance of Bax/Bcl-2. This led to cleavage and activation of caspase- 3 and PARP, and then induced apoptosis of Raw264.7 cells. In addition, either acute or chronic applications of LN were sufficient to disrupt the structure of the hepatic portal vein and reduce the number of Kupffer cells in mice.

Conclusions: LNs could induce apoptosis of macrophages through a mitochondrial-dependent pathway.

Keywords: Lipidic nanocarrier, cell death, macrophages in liver, Bax/Bcl-2, Caspase-3

\section{Introduction}

Lipid-based drug delivery systems, sometimes referred to as lipidic carriers, are widely used today because they have the ability to incorporate lipophilic and hydrophilic molecules and stabilize them in vitro and in vivo. ${ }^{1,2}$ In addition, it has been repeatedly shown that the encapsulation of bio-active molecules inside these carrier systems can enhance the bioavailability, efficacy, and safety of poorly soluble drugs. ${ }^{3-5}$ Thus, this technology has grown to be more and more widely utilized in the production and use of cosmetics, foods/nutritional preparations, nutraceuticals, and pharmaceuticals. The efficacy and toxicity of these lipidic carriers have been largely related to their physiochemical properties, which in turn is determined by their composition and formulation. ${ }^{6}$ To date, many different types of lipidic carriers have been introduced, including liposomes, nano-liposomes, archaeosomes, lipid nanoparticles (LNs), and tocosomes. Among them, LNs have become useful for drug delivery as a result of their consistent composition and ease of use. ${ }^{7}$ LNs are prepared largely with emulsification processes from
Correspondence: Wei Kevin Zhang; HeBin Tang

School of Pharmaceutical Sciences, SouthCentral University for Nationalities,

Room 13-218, No. 182 Minzu Avenue,

Wuhan 430074, People's Republic of

China

Tel +86I 3995697887

Email wkzhang8I@I39.com;

hbtang2006@mail.scuec.edu.cn
International Journal of Nanomedicine 2019:14 3283-3295

3283 
an oil phase (eg, dietary oils), aqueous phase (water or aqueous solutions), and interfacial phase (where tiny amounts of surfactant or other amphiphilic molecules are located). Thus, the physical and chemical properties of LNs are quite variable, depending on their compositions. ${ }^{8}$ Researchers are trying to overcome the current limitations for administration and widen the possible applications of LNs, and recent advances have led to improvements in the delivery of siRNA ${ }^{9}$ and $m R N A,{ }^{10}$ the dermal delivery of retinoic acids, ${ }^{11}$ pulmonary application, ${ }^{12}$ and treatment of chronic wounds by LNs. ${ }^{13}$

We recently reported another possible application of LNs: enhancing the tumorigenicity of diethylnitrosamine and engendering liver cancer in mice, ${ }^{14}$ which could potentially result in a useful animal model of hepatic carcinoma. However, during these experiments, we noticed that a certain level of apoptosis was occurring in Kupffer cells in the murine liver samples treated only with the LN vehicle via the TUNEL staining.

Kupffer cells are a type of tissue-resident macrophage usually located in liver sinusoids. ${ }^{15}$ They perform a critical role in the innate immune response of the mononuclear phagocytic system in the liver. ${ }^{16}$ On one hand, pathogens entering the liver from the portal or arterial circulation could be phagocytized and inactivated. On the other hand, pro-inflammatory particles and materials from the gastrointestinal tract could also be phagocytized by the Kupffer cells. This makes Kupffer cells particularly important for the development of acute and chronic liver injuries through various etiologies. ${ }^{17}$ As a result, malfunction of the Kupffer cells is associated with many disease states, including drug-induced liver injury, ${ }^{18}$ toxin-induced fibrosis, ${ }^{19}$ and chronic hepatitis resulted from either alcoholic or nonalcoholic fatty liver disease. ${ }^{20}$

Currently, apoptotic effects of solid LNs on macrophages and Kupffer cells have not been elucidated. Therefore, in this study, we investigated whether solid LNs affect Kupffer cells, and what mechanisms induce apoptosis of macrophages, using the macrophage cell model raw264.7 in vitro and the liver samples of mice in vivo.

\section{Materials and methods}

\section{Reagents}

Tween-80 (Amresco, \#0442, USA) and Tween-20 (Amresco, \#0777) were purchased. Lecithin from egg yolk was provided by Sinopharm Chemical Reagent
(\#6901933, Shanghai, China). Sesame oil was obtained from Blessing Mill (Wuhan, China). DMEM (\#10569044) and FBS (\#12484-010) were purchased from Gibco (Thermo Fisher Scientific, Waltham, MA, USA). MTT (\#M2128), dimethyl sulfoxide (DMSO, \#D5879) of analytical reagent grade and Glycerol ReagentPlus (GC grade, \#G7757) were obtained from Sigma-Aldrich (St Louis, MO, USA).

\section{Preparation and characterization of LNs}

A high-pressure microfluidics technique was used for the formulation of LNs. Briefly, the lipid phase, consisting of $7.52 \mathrm{~mL}$ of sesame oil and $0.240 \mathrm{~g}$ of lecithin from egg yolk, was dissolved in $30 \mathrm{~mL}$ ethanol and heated to $60^{\circ} \mathrm{C}$. After removal of the solvent by rotary evaporation, $32 \mathrm{~mL}$ of preheated water (containing $0.9 \mathrm{~mL}$ of glycerol and 0.4 $\mathrm{mL}$ Tween-80) was added gradually to the hot and molten lipid sample and was magnetically stirred gently for 10 mins. A coarse oil-in-water emulsion was formed by highspeed shearing via a Fluko FA25 homogenizer (Fluko Equipment Shanghai Co., Ltd., Shanghai, China) at $10,000 \mathrm{rpm}$ for 2 mins. The coarse emulsion was further homogenized for six cycles at 1,000 bars, using $\mathrm{M}$ 100PCE, a high-pressure microfluidics device (Microfluidics Corporation, Westwood, MA, USA). The hot dispersion was cooled down to $4^{\circ} \mathrm{C}$ and sterilized by passing it through a $0.45 \mu \mathrm{m}$ cellulose acetate filter. Finally, the resulting material was stored in brown glass vessels at $4^{\circ} \mathrm{C}$.

\section{Measurement of particle size and zeta potential of LNs}

Particle size and zeta potential were measured by a Zetasizer Nano ZS90 (Malvern Instruments, Malvern, UK). Particle size (as hydrodynamic diameter, in $\mathrm{nm}$ ) and the polydispersity index were determined by diluting $10 \mu \mathrm{L}$ of the LNs with $4 \mathrm{~mL}$ of ultrapure water. To determine the zeta potential, the LNs were diluted with ultrapure water until the conductivity of the dilute suspension was in the range of $40-50 \mu \mathrm{S} / \mathrm{cm}$. All measurements are presented as summaries of triplicates.

\section{Field emission-scanning electron microscopy (FE-SEM)}

The morphology of the LNs was observed by an FE-SEM (SU8010, HITACHI, Japan), for which a $100 \mu \mathrm{L}$ aliquot of the LN preparation was diluted with deionized water to a 
volume of $2 \mathrm{~mL}$ and sonicated for $1 \mathrm{~min}$. SEM samples were prepared by placing one drop of the diluted LNs onto a carbon-coated copper grid (400 mesh; Beijing Xinxing Braim Technology Co., Ltd, Beijing, People's Republic of China) and allowing adsorption for 10 mins. Excessive liquid was then blotted with a filter paper. After drying naturally for $1 \mathrm{hr}$, the dried specimen was observed with SEM at an acceleration voltage of $35 \mathrm{kV}$.

\section{Cell culture and MTT assay}

Mouse monocyte-macrophages (Raw264.7, \#GDC0143), human liver cancer cells (HepG2, \#GDC141) and normal human liver cells (L02, \#GDC079) were purchased from the China Center for Type Culture Collection (Wuhan, China) and were maintained as described by the vendor's instructions. The cytotoxicity of the LNs in Raw264.7 cells was assessed by the MTT assay. Briefly, cells $(1 \times$ $10^{5}$ cells $/ \mathrm{mL}$ ) were seeded into 96 -well plates in DMEM medium supplemented with $10 \%$ FBS, and then treated with growth medium containing either $250 \mu \mathrm{g} / \mathrm{mL}$ LNs for the indicated periods of time or indicated concentrations of LNs for $5 \mathrm{hrs}$. After that, $20 \mu \mathrm{L}$ of MTT solution $(5 \mathrm{mg}$ / $\mathrm{mL}$ ) was added to each well and the plate was incubated at $37^{\circ} \mathrm{C}$ for $4 \mathrm{hrs}$. Subsequently, DMSO was added and the absorbance was measured at $570 \mathrm{~nm}$ using a microplate reader (Infinite 200 PRO; Tecan Group Ltd., Maennedorf, Switzerland).

\section{Detection of apoptosis}

Cultured Raw264.7 cells were seeded at a density of $1 \times$ $10^{6}$ cells per well in a six-well plate. After treatment for the indicated periods of time or concentrations, the cells were washed with PBS twice and then aspirated with $1 \mathrm{~mL}$ PBS. Then, they were suspended in $400 \mu \mathrm{L}$ of binding buffer and $5 \mu \mathrm{L}$ Annexin-V-FITC for 15 mins at $4^{\circ} \mathrm{C}$. Finally, $10 \mu \mathrm{L}$ propidium was added for 5 mins at $4^{\circ} \mathrm{C}$, and detected by flow cytometry (Guava easyCyte System; Merck, Darmstadt, Germany) or observed by fluorescence microscope (Nikon Inc., Tokyo, Japan). The Annexin-VFITC/PI apoptosis detection kit for flow cytometry was purchased from BestBio (\#BB-4101-50T, Shanghai, China).

\section{Evaluation of mitochondrial membrane depolarization}

The depolarization of mitochondrial membranes was detected with a mitochondrial membrane potential assay kit with JC-1 according to the manufacturer's protocol (\#40606ES60; Yeasen,Shanghai, China). JC-1 is a potentiometric dye that exhibits a membrane potential-dependent loss as JC-1 aggregates (polarized mitochondria) transition to JC-1 monomers (depolarized mitochondria). The loss of membrane potential is indicated by a shift of the fluorescence emissions from red to green. The levels of the cells' fluorescence intensity were analyzed by flow cytometry (Guava easyCyte System; Merck) using an excitation wavelength of $488 \mathrm{~nm}$ and emission wavelengths of 530 and $585 \mathrm{~nm}$ for green and red fluorescence, respectively.

\section{Transmission electron microscopy (TEM) observations on Raw264.7 cells}

The Raw264.7 cells exposed to LNs under various conditions were harvested and fixed in 1\% osmic acid, and were subsequently dehydrated in a series of graded alcohol solutions. Then, $60 \mathrm{~nm}$ sections were cut and stained with uranyl acetate and lead citrate and mounted on a carbon-coated copper grid (400 mesh; Beijing Xinxing Braim Technology Co.). After that, cells were observed via TEM using a Hitachi HT7700 unit (Hitachi Corp., China).

\section{Western blotting}

After incubation with a $250 \mu \mathrm{g} / \mathrm{mL}$ concentration of the LNs for $0,1,2,3,4$, or $5 \mathrm{hrs}$, cells were lysed with a protein lysis buffer (\#P0013; Beyotime, Nanjing, China). The total protein concentration was measured by the Lowry method. Equal amounts of protein were fractionated using $12.5 \%$ SDS gels and transferred to polyvinylidene difluoride membranes (\#IPVH00010; Millipore Corporation, Billerica, MA, USA). After being blocked with $5 \%$ non-fat dry milk, the membranes were incubated with an anti-caspase 3 antibody $(1: 1,000$, Cell Signaling Technology, \#9662S), anti-PARP antibody (1:1,000, Cell Signaling Technology, \#9542S), anti-Bax antibody (1:1,000, Cell Signaling Technology, \#5023S), anti-Bcl-2 antibody (1:1,000, Cell Signaling Technology, \#3498S), or anti-actin antibody $(1: 1,000$, Cell Signaling Technology, $\# 4970 \mathrm{~S}$ ) at $4^{\circ} \mathrm{C}$ overnight. After removing the primary antibodies, the membranes were washed three times for 5 mins with TBST (Tris-buffered saline, $0.1 \% \mathrm{v} / \mathrm{v}$ Tween20) solution and followed by exposure to secondary antibodies $(1: 2,000$, goat anti-rabbit or goat anti-mouse, Abbkine, USA, \#A21010, \#A21020) for $1 \mathrm{hr}$ at room 
temperature. Finally, after washing, protein bands on the membranes were visualized by a developer and fixing solution. The protein bands were quantified using the ImageJ software and were analyzed and plotted with Instat software (GraphPad Prism 7, La Jolla, CA, USA)

\section{Animal care}

The care and use of animals and experimental protocols for this study were carried out according to the Guide for Animal Experimentation, South-Central University for Nationalities and the Committee of Research Facilities for Laboratory Animal Sciences, South-Central University for Nationalities, China. The protocols were approved by the Committee on the Ethics of Animal Experiments of the South-Central University for Nationalities, China (permit number: 2018-SCUEC-AEC011). All efforts were made to minimize suffering including anesthesia by $\mathrm{CO}_{2}$ before sacrifice.

\section{In vivo experiments with $\mathrm{LNs}$}

Fifty-four male Kunming mice (8 weeks old, 20-22 g) were acclimatized for 7 days under specific-pathogenfree conditions before experimentation. The animals were kept on a basal diet in a temperature-controlled animal room $\left(22-25^{\circ} \mathrm{C}\right)$ with a $12 \mathrm{hrs}$ light-dark cycle for at least 1 week before any experiments were performed. In the acute experiments, the animals were randomly separated into three groups of 10 mice each: control group, a low-dose group, and a high-dose group. The low-dose group and high-dose group LN-groups received $10 \mathrm{mg}$ / $\mathrm{kg}$ and $30 \mathrm{mg} / \mathrm{kg} \mathrm{LN}$ orally (as a volume of $5 \mu \mathrm{L} / 10 \mathrm{~g}$ for both groups), respectively, every 12 hrs for 3 days. The control group mice received normal saline $(5 \mu \mathrm{L} / 10 \mathrm{~g})$. In the chronic experiments, the control group $(n=12)$ and the LNs-treated group $(n=12)$ both received normal saline and $10 \mathrm{mg} / \mathrm{kg} \mathrm{LN}$ orally, once every week for 20 weeks. One hour before sacrifice, six mice from each group in the LNtreated group were treated by gastric application of ink for subsequent analysis of ink phagocytosis. Upon sacrifice, their livers were immediately collected and fixed for histology and protein analysis.

\section{Histopathological analysis of liver tissues}

Fresh liver tissue samples were fixed in 10\% neutral formalin, embedded in paraffin, cut into $2 \mu \mathrm{m}$ slices, and stained with hematoxylin and eosin (H\&E) or hematoxylin alone by standard techniques. Histopathologic examination of the liver sections was conducted using a Nikon 50i light microscope (Nikon Inc.).

\section{Immunofluorescence analysis of liver tissues}

The samples were deparaffinized, hydrated and blocked with $3 \%$ hydrogen peroxide for 15 mins. Specimens were then subjected to antigen retrieval by immersing them in $0.01 \mathrm{M}$ boiling citrate buffer and heating in a microwave oven for 1 min. After blocking with 5\% BSA (Cat: 36106ES25; Yeasen) for 40 mins, the sections were incubated overnight at $4^{\circ} \mathrm{C}$ with primary antibodies against lysozyme (1:1,000, ab108508; Abcam, Cambridge, UK). After incubation with fluorescein-conjugated IgG secondary antibodies (1:200, CWBio, CW0161S, Beijing, China) at $37^{\circ} \mathrm{C}$ for 40 mins. DAPI (Cat: 17985-50; Electron Microscopy Sciences, Hatfield, PA, USA) was used as a nuclear counterstain in the tissue sections, which were mounted using coverslips.

\section{Statistical analysis}

Results are shown as means \pm SEM. The statistical analyses were performed by one-way ANOVA followed by Tukey's test as indicated in the text using Instat software (GraphPad Prism 7). A $P$ value $<0.05$ was considered statistically significant.

\section{Results \\ Characterization of LNs}

The characteristics (including the size, zeta potential and morphology) of LNs are shown in Figure 1. The particle size of the LNs was approximately $212.8 \mathrm{~nm}$, and their zeta potential was around $-28.7 \mathrm{mV}$ (Figures $1 \mathrm{~B}$ and $\mathrm{C}$ ). The FE-SEM studies clearly indicated that the LNs were spherical in shape and had a smooth surface (Figure 1D).

\section{Apoptosis of raw264.7 cells was observed after application of LNs}

To evaluate the effect of LNs on the viability of the Raw264.7 cells, they were incubated either with different concentrations (Figure 2B) of LNs or for different time intervals (Figure 2A). After incubation, cell viability was evaluated using a MTT assay. Compared to the control group, viability rates of cells treated with $250 \mu \mathrm{g} / \mathrm{mL} \mathrm{LNs}$ for $0,1,2,3,4$ and $5 \mathrm{hrs}$ were $100.0 \pm 10.8 \%, 90.0 \pm 11.8 \%$, $59.0 \pm 8.5 \%, 12.0 \pm 4.0 \%, 0.6 \pm 0.4 \%$ and $7.0 \pm 13.9 \%$, respectively (Figure 2C). As shown in Figure 2D, the viability 


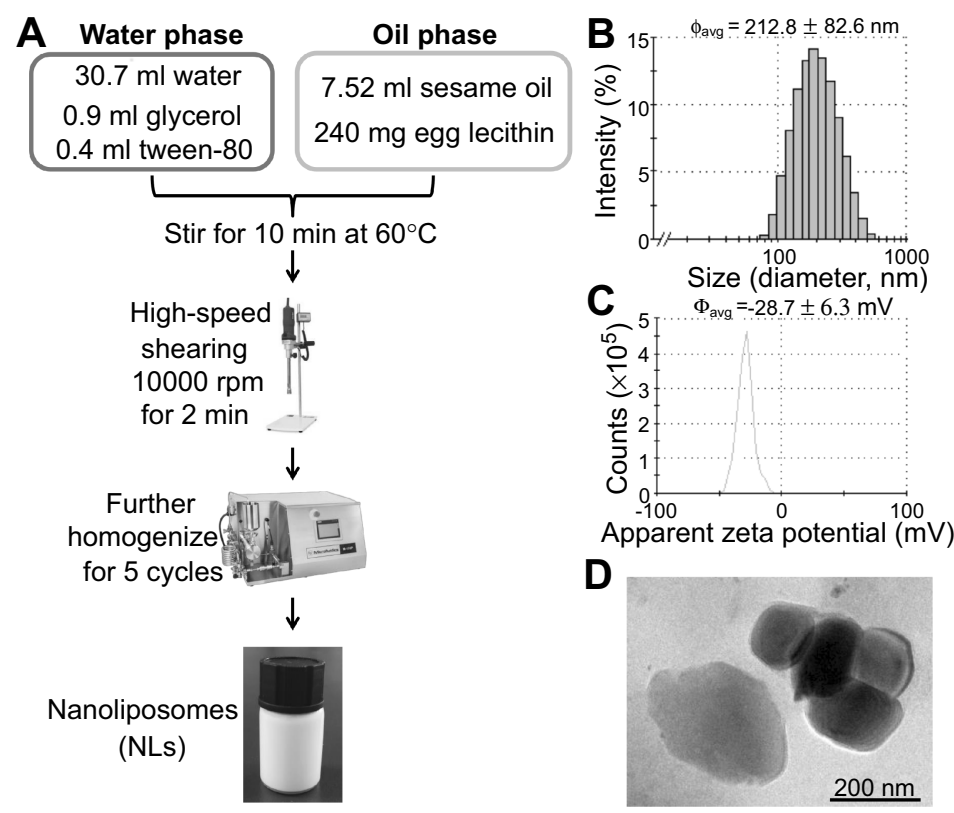

Figure I Fabrication and characterization of lipid nanoparticle (LNs). (A) General procedure of fabricating LNs. (B) Particle sizes of our laboratory-made LNs. (C) Apparent zeta potential of our laboratory-made LNs. (D) Scanning electron microscope images of the nanoparticles. Scale bar, $200 \mathrm{~nm}$.

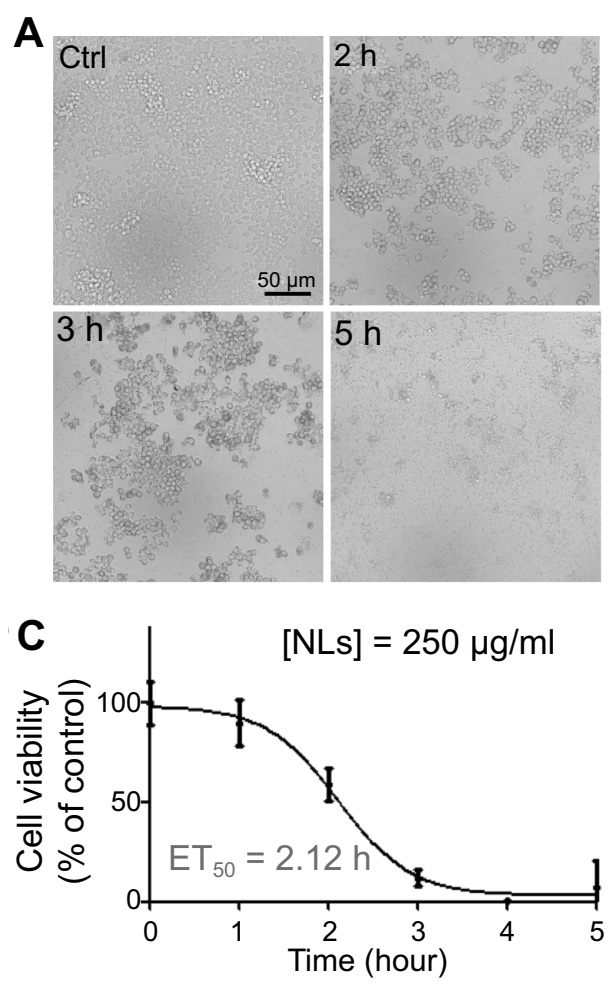

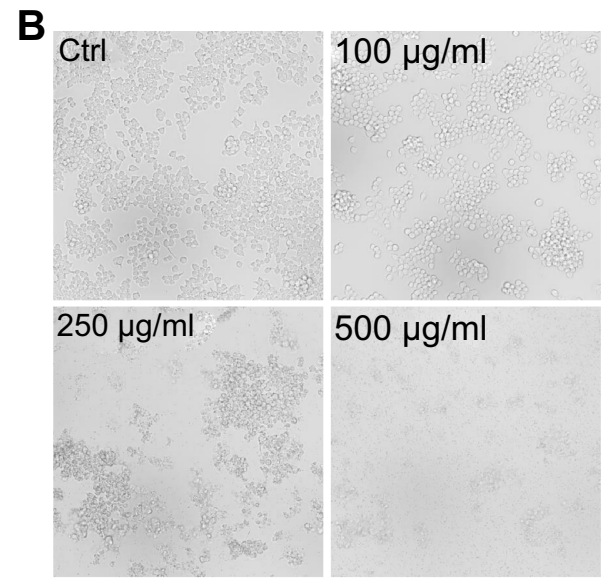

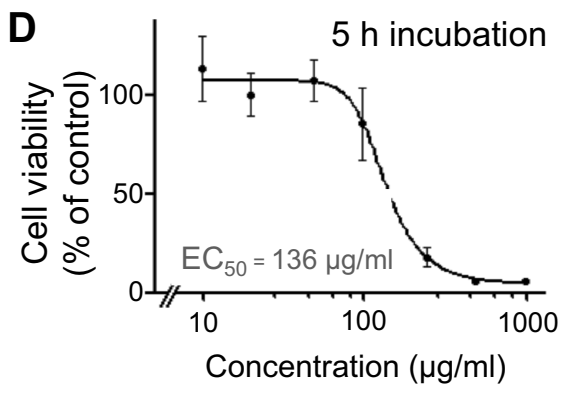

Figure 2 Treatment of lipid nanoparticles (LNs) reduced the viability of Raw264.7 macrophages in a dose- and time-dependent manner. (A) Representative figures of Raw264.7 cells after treatment with $250 \mu \mathrm{g} / \mathrm{mL} \mathrm{LNs}$ for various periods. Scale bar, $50 \mu \mathrm{m}$. (B) MTT assay revealed the ET 50 of LNs was 2.12 hrs. Each point represents an average of duplicates from three individual experiments. (C) Representative figures of Raw264.7 cells after treatment with various concentrations of $L N s$ for 5 hrs. Scale bar, $50 \mu \mathrm{m}$. (D) MTT assay revealed the $\mathrm{EC}_{50}$ of $\mathrm{LNs}$ was $136 \mu \mathrm{g} / \mathrm{mL}$. Each point represents an average of duplicates from three individual experiments.

rates of cells treated with $0,100,250$ and $500 \mu \mathrm{g} / \mathrm{mL} \mathrm{LNs}$ for $5 \mathrm{hrs}$ were $100.0 \pm 8.9 \%, 85.9 \pm 18.4 \%, 18.1 \pm 4.6 \%$ and $5.7 \pm 0.2 \%$.
Moreover, we also evaluated the apoptotic effect of LNs in the Raw264.7 cells using Annexin-V-FITC/PI double staining and detected the results using fluorescent 
microscope and flow cytometry. As shown in Figures $3 \mathrm{~A}$ and C, Raw264.7 cells were exposed to $250 \mu \mathrm{g} / \mathrm{mL}$ LNs. The percentage of surviving cells decreased significantly in a time-dependent manner, and the percentage of latestage apoptosis cells increased significantly after 2 hrs. The ratio of early to late apoptosis was maximal after 5 hrs. We also measured apoptosis occurring in the Raw264.7 cells under various concentrations of LNs, ranging from 100 to $500 \mu \mathrm{g} / \mathrm{mL}$. As shown in Figures 3B and $\mathrm{D}$, apoptotic and necrotic cells increased in the LN samples in a dose-dependent manner. The ratio of apoptoticto-viable cells was very high after exposure to a concentration of $500 \mu \mathrm{g} / \mathrm{mL}$.

\section{The mitochondria of raw264.7 were disrupted by $\mathrm{LN}$ treatment}

The mitochondria in Raw264.7 cells were studied by electron microscopy. LN treatment can destroy mitochondrial structure, and the degree of damage increases in a doseand time-dependent manner as shown in Figure 4A. LNs induced depolarization of the mitochondrial membrane potential as well. The effect of LNs on the mitochondrial membrane permeability of Raw264.7 cells was evaluated using JC-1 fluorescent dye. As shown in Figure 4B, following treatment of the LNs, the intensity of the green fluorescence increased, while the intensity of the red fluorescence decreased. After the cells were treated with 250 $\mu \mathrm{g} / \mathrm{mL}$ of $\mathrm{LN}$, the green fluorescence was increased by $28.10 \%$ and $28.50 \%$ at 4 and 5 hrs, respectively, compared to a $5.92 \%$ increase in the untreated control group.

\section{LNs induced apoptosis via disruption of the balance between bax and bcl-2}

Western blot analysis was employed to determine the effect of the LNs on the expression of proteins involved in the apoptotic pathway. Figure 5A and B depicts the changes that occurred after treatment with the LNs, with the expression level of Bax having been up-regulated, while at the same time Bcl-2, caspase-3 and PARP were down-regulated. Moreover, after exposure to the LNs at a concentration of $250 \mu \mathrm{g} / \mathrm{mL}$ for a period of $3 \mathrm{hrs}$, the cleaved caspase-3 and PARP bands were observed and

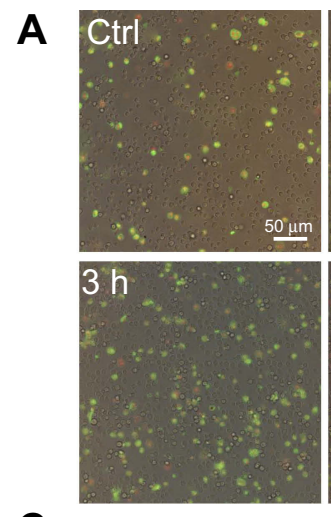

C

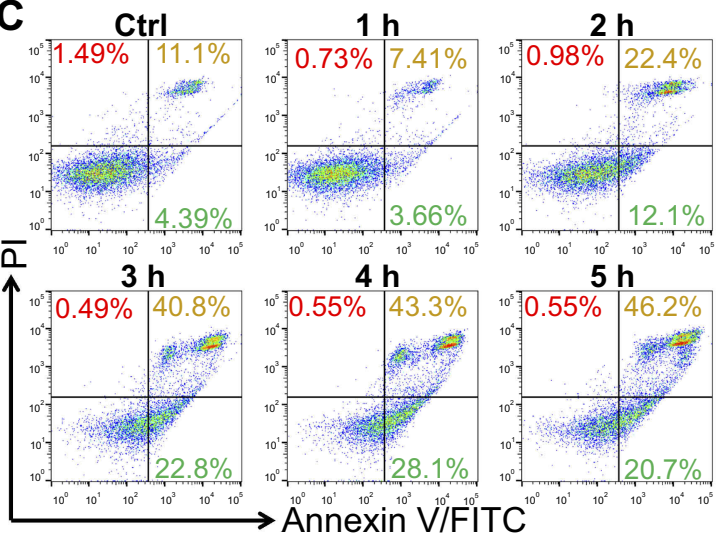

B
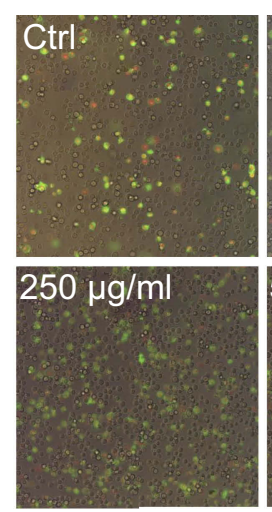

D
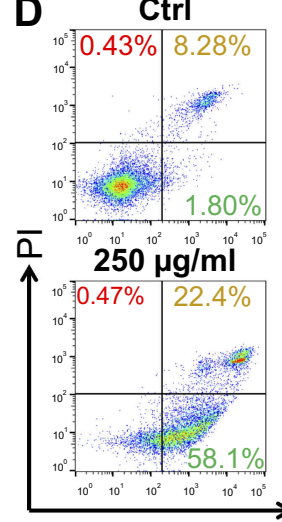
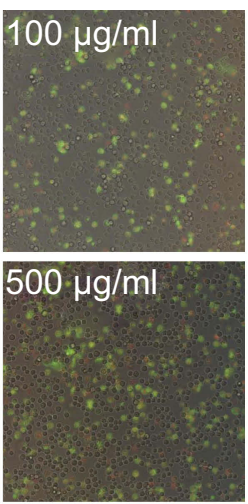

$100 \mathrm{\mu g} / \mathrm{ml}$

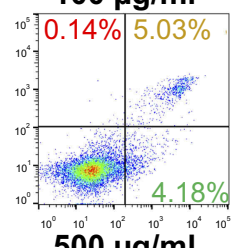

$\mathbf{5 0 0} \mathbf{\mu g} / \mathrm{ml}$

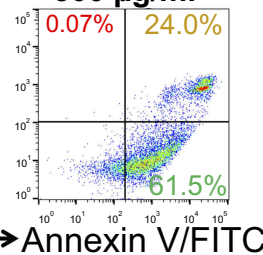

Figure 3 Lipid nanoparticle (LN) induced apoptosis of Raw264.7 cells in vitro. Apoptotic cells were stained with Annexin-V-FITC and PI and observed by fluorescence microscope (magnification $\times 200$ ) after treatment $(\mathbf{A})$ for various periods or $(\mathbf{B})$ with different concentrations. Green fluorescence indicated cells underwent an early phase of apoptosis while red fluorescence indicated late phase apoptosis. Scale bar, $50 \mu \mathrm{m}$. Flow cytometry was also used for separating phases of apoptosis after treatment of LNs either (C) for various periods or (D) with different concentrations. 

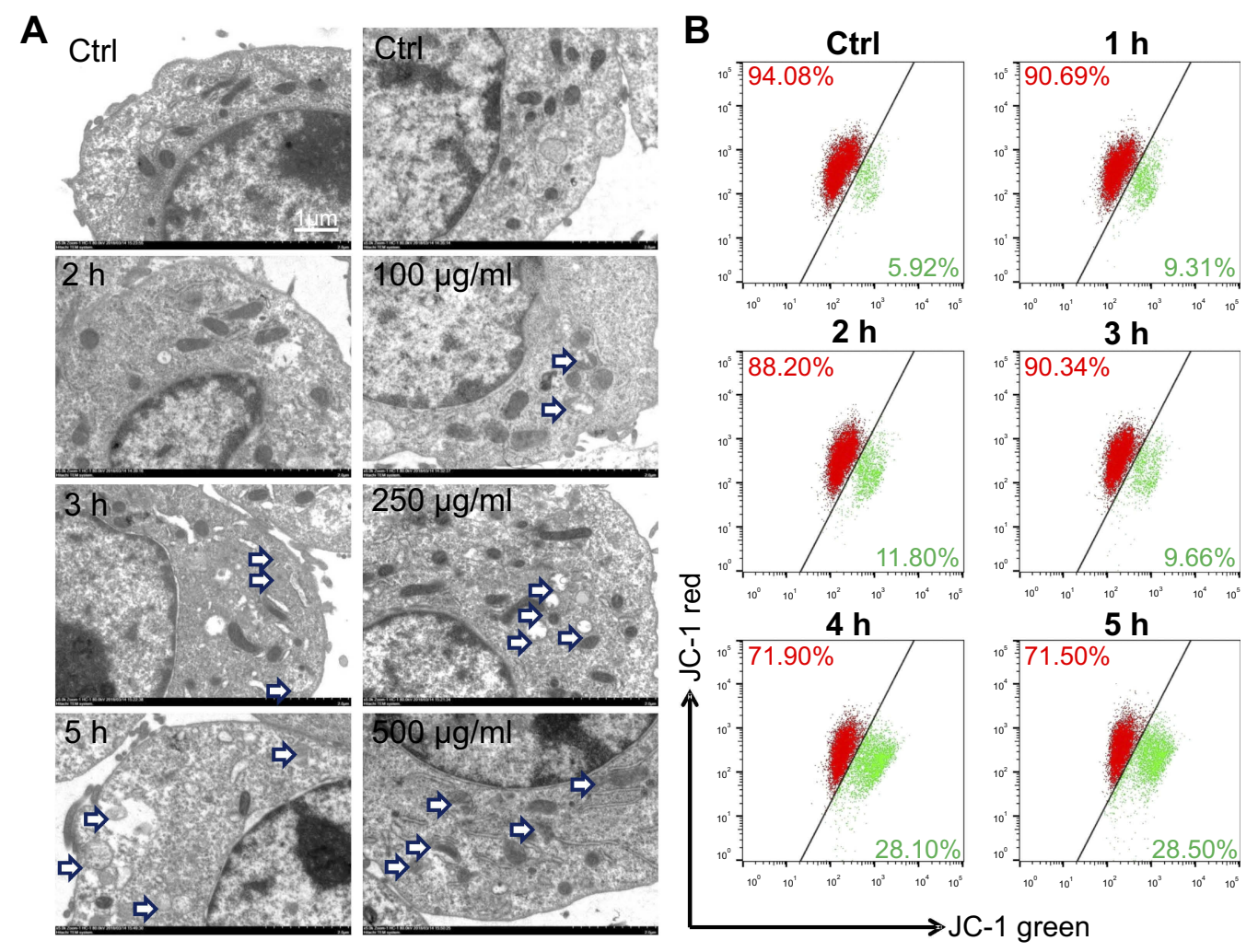

Figure 4 Lipid nanoparticle (LN) treatment disrupted mitochondria of Raw264.7 cells. (A) Swelled or broken mitochondria were observed in Raw264.7 cells after the application of LNs (magnification $\times 5,000$ ). The blue arrow indicates swollen or broken mitochondria. (B) Flow cytometry revealed a shift of the mitochondrial membrane from polarized state (red fluorescence) to depolarized state (green fluorescence) after application of LNs in a time-dependent manner.
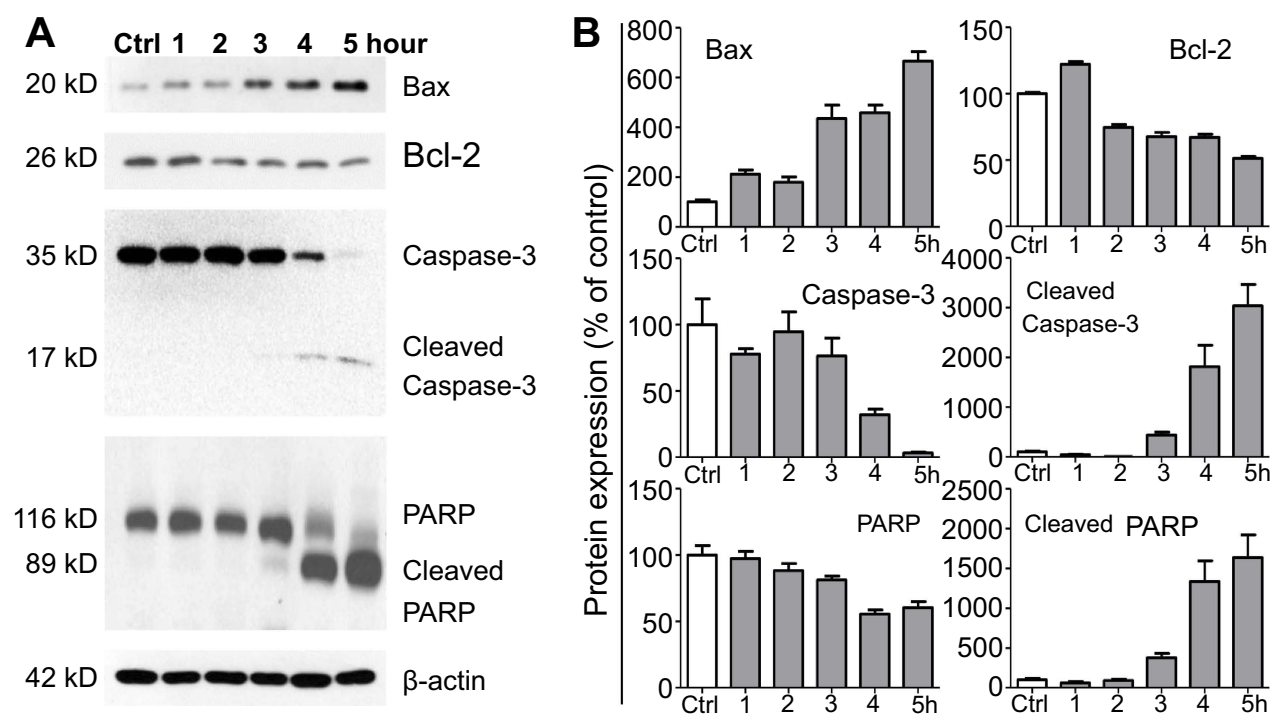

Figure 5 Lipid nanoparticle (LN) treatment varied expression levels of apoptosis-related proteins in Raw264.7 cells. (A) Representative western blot images and (B) summary of expression changes among Bax, $\mathrm{Bcl}-2$, Caspase-3, and PARP. The expression of $\boldsymbol{\beta}$-actin was used as a loading control. Each bar represents an average of four independent experiments. 
noted to have been impacted afterwards. These data indicated that the apoptotic effect of LNs on macrophages was exerted by disrupting the balance between Bax and Bcl-2.

\section{Effects of $L N$ treatment on kupffer cells in mice}

To further evaluate the effect of LNs in vivo, acute and chronic experiments were conducted in mice. After oral application of LNs to the mice as indicated (Figures 6A and $\mathrm{A}), \mathrm{H} \& \mathrm{E}$ staining was used to detect histological changes in the liver after both short-term (Figure 6) and long-term (Figure 7) exposure. As shown in Figure 6B, the structure of the tissues near the portal veins in the low-dose $(10 \mathrm{mg} / \mathrm{kg})$ and high-dose $(30 \mathrm{mg} / \mathrm{kg})$ groups was more disordered than that in the control group. Similar phenomena were also observed after chronic exposure to the LNs (Figure 7B). The Kupffer cells in the liver were measured by immunofluorescence staining and the results showed that either type of exposure would decrease lysozyme-positive cells, suggesting that both acute and chronic application of LNs could cause loss of Kupffer cells. The same trend was also noted in the results of the ink phagocytosis experiments (Figures 7B and D).

\section{Discussion}

LNs have drawn attention from researchers since they were first developed, as a result of their unique physical, chemical and biological properties. Today, there is elevated public awareness of these agents, accompanying their increasing usage. In the present study, we observed the apoptotic effects of one kind of LNs on Kupffer cells, and concluded that these effects were exerted via a mitochondrial pathway. However, several points need to be clarified:

First, the spherical shape and size of our LN followed a narrow normal distribution pattern that was similar to that observed in our previous investigations on LNs. ${ }^{14,21,22}$ The negative zeta potential of the LNs could be a result of the forming materials that we used, and could also play an important role during their internalization into cells (or at least the attachment of LNs at the outer membrane of the cells), since the exterior side of the cell membrane usually had a positively charged layer of ion (mostly aqueous $\mathrm{Na}^{+}$
A

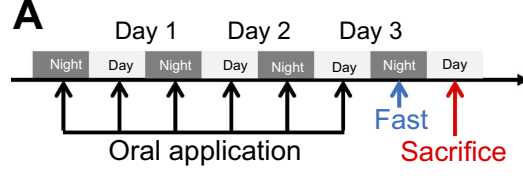

Acute application protocol

\section{B}

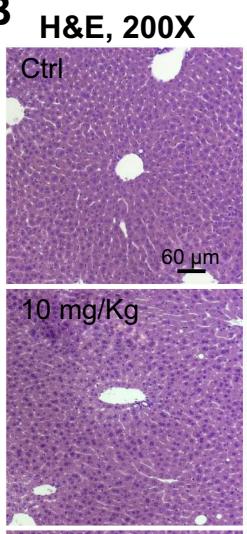

H\&E, $400 X$

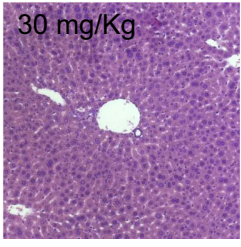

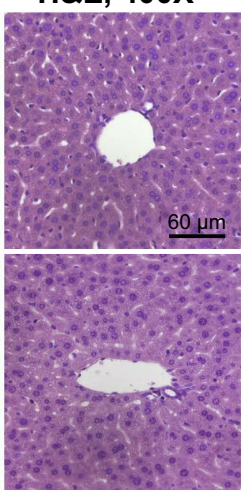

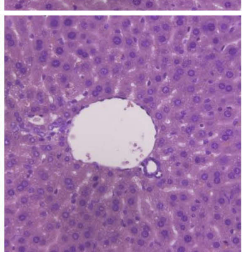

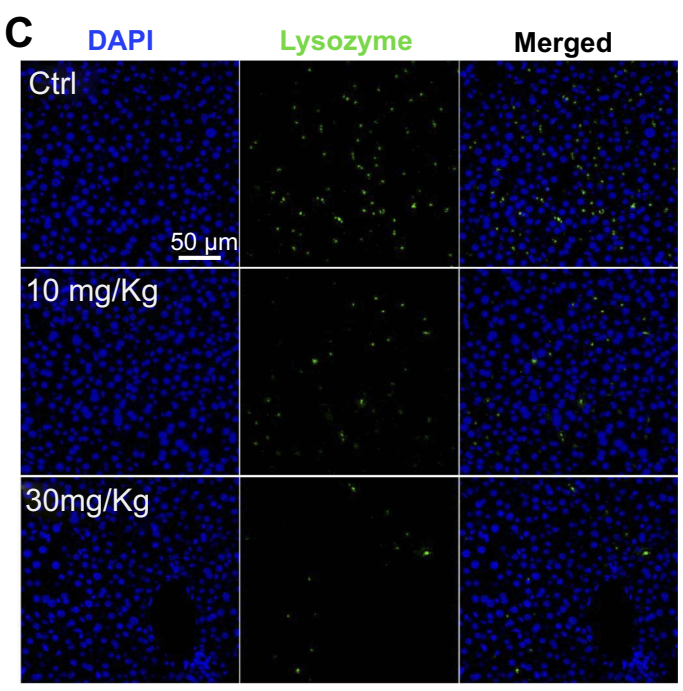

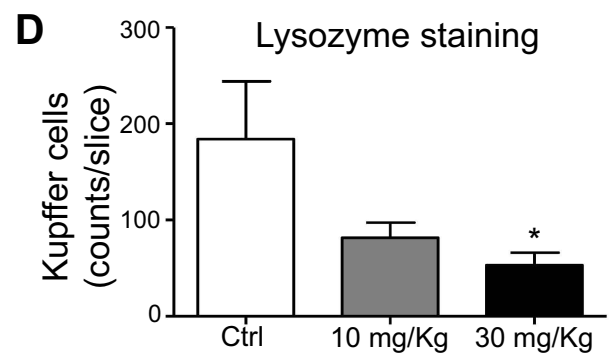

Figure 6 Short-term application of lipid nanoparticles (LNs) revealed its acute toxicity on Kupffer cells in liver specimens from mice. (A) Detailed protocol of LN shortterm oral application to mice. (B) H\&E staining of liver specimen under the application of different concentrations of LNs. Scale bar, $60 \mu \mathrm{m}$. (C) Representative images and (D) summary of immunofluorescence staining of lysozyme (marker of resident macrophages in liver). Scale bar, $50 \mu \mathrm{m}$. *represents significant difference with $P<0.05$. 

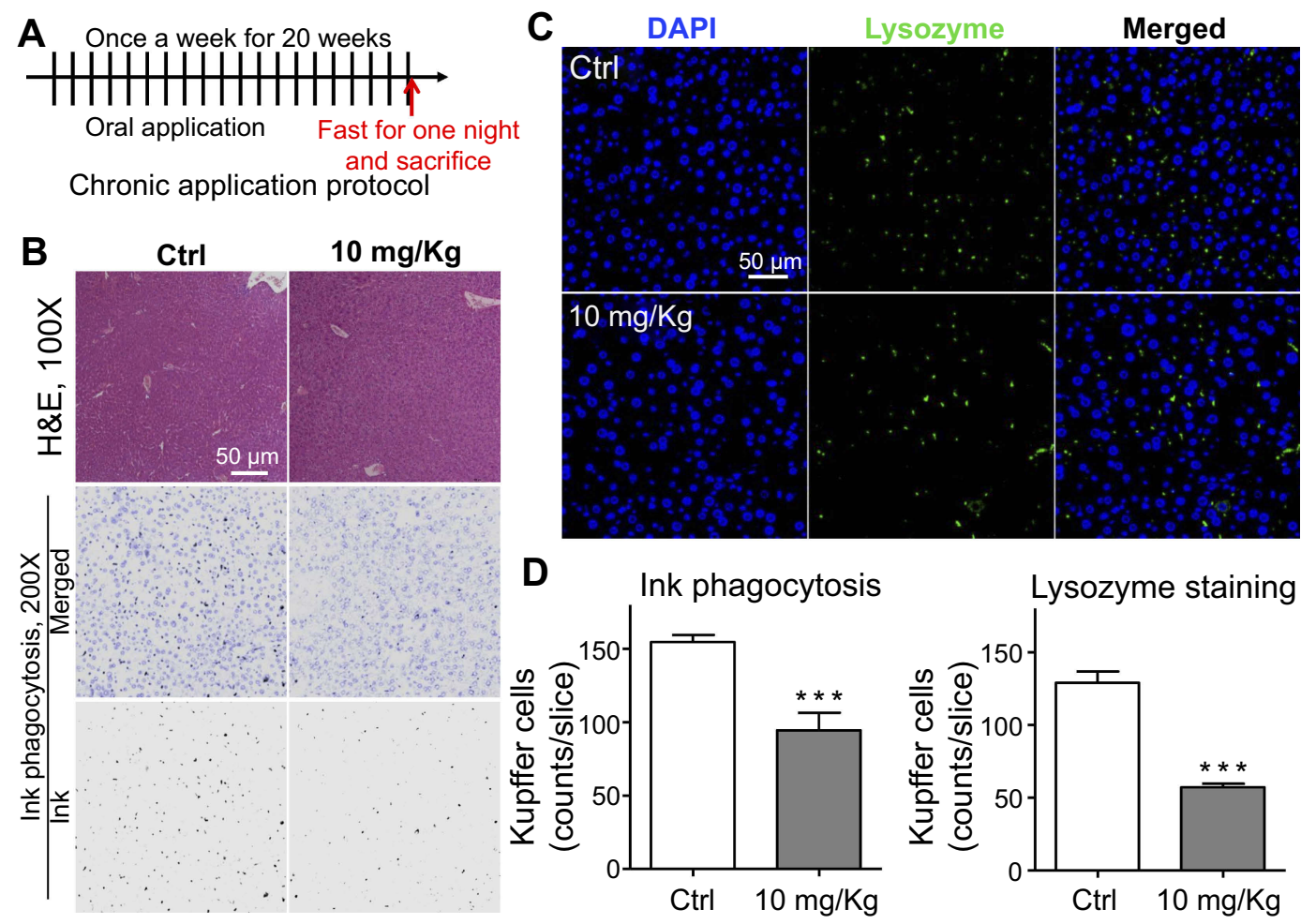

Figure 7 Chronic toxicity on Kupffer cells observed after long-term application of lipid nanoparticles (LNs). (A) Detailed protocol of LN long-term oral application to mice. (B) H\&E staining of liver specimen under the application of LNs. Scale bar, $50 \mu \mathrm{m}$. (C) Representative images and (D) summary of immunofluorescence staining of lysozyme (marker of resident macrophages in liver). Scale bar, $50 \mu \mathrm{m}$. ***represents significant difference with $P<0.00 \mathrm{I}$.

ions). Considering the role of macrophages in phagocytosis, this negative zeta potential could actually facilitate uptake of LNs into the Kupffer cells.

Secondly, based on our current research, this toxic effect of LNs was restricted to Kupffer cells, and was not observed in other types of cells in the liver, especially hepatocytes (Figure S1 and Table S1). Hence, no difference was observed on the values and the ratio of aspartate transaminase and alanine transaminase in our mouse studies, which presumably indicated that the LNs could affect the Kupffer cells, but not cause acute damage to the hepatic parenchyma during the application period. This may be explained partly as a result of the difference in endocytosis of various cell types. Macrophages possess the ability to engulf particles and engage in phagocytosis as a primary function. In contrast, hepatocytes are capable of performing some endocytosis. But this activity is not comparable to that of macrophages with respect to its rate or scale of particle sizes during material internalization. Thus, taking into consideration the uptake facilitation provided by the negative zeta potential of the LNs, it would not be astonishing if these LNs manifested higher levels of toxicity on macrophages than on the ordinary hepatocytes. It is also interesting, that after the application of much higher concentrations of LNs, both the HepG2 and L02 cell cultures also displayed cell losses (Figure $\mathrm{S} 1$ ), suggesting potential toxicity of high doses of LNs on the hepatocytes. Nevertheless, long-term applications of LNs could still affect the normal physiological functions of liver as a result of persistent apoptosis and loss of the Kupffer cells. Moreover, we observed toxicity from our LNs on Raw264.7 cells, a cell model for macrophages. LNs could also induce apoptosis in other types of tissuespecific macrophages, including alveolar macrophages in pulmonary tissues, microglia in brain and spinal cord, sinus histiocytes in lymph nodes, and intestinal macrophages in the GI tract. Although these different types of macrophages show variable functions and activation patterns, malfunction or loss of these key cells of the immune system could cause severe abnormalities. ${ }^{23}$ Moreover, the $\mathrm{EC}_{50}(136 \mu \mathrm{g} / \mathrm{mL})$ may reflect an upper limit of safe application of LNs in vitro, whereas the $30 \mathrm{mg} / \mathrm{kg}$ dose could be treated as an upper limit for safe acute application of LNs in vivo.

In addition, the detailed functions of Kupffer cells in the liver are being viewed as more and more important. On 
one hand, Kupffer cells are located in the hepatic sinusoids, a location that allows them to phagocytize pathogens entering the liver from either the arterial circulation or the GI tract via the portal circulation. On the other hand, Kupffer cells are highly involved in the clearance of apoptotic cells from the hepatic parenchyma. ${ }^{24}$ Prior investigations have suggested that many hepatic diseases are associated with impaired activity of the Kupffer cells, including drug-induced liver injury, toxin-induced fibrosis and chronic inflammations during hepatitis. Thus, these cells play critical roles in the progression of liver injury and repair. Based on our current findings, the toxic effect of LNs could provide a simple way to functionally ablate Kupffer cells in normal liver, and thus the LNs could serve as a tool to further our understanding of the possible functions of Kupffer cells and how they are involved in normal physiology. Using experimental animal models, LNs could be easily introduced at any point in the progression of liver pathology and thus be used to clarify the possible regulatory roles of Kupffer cells during a variety of stages of disease progression. Furthermore, investigations could be conducted on the toxicity of LNs with different sizes or compositions of the particles in order to search for potential-specific tools for ablating macrophages of various origins and/or in different polarization states.

Additionally, major components of LNs were separately applied to the Raw264.7 cells in order to determine which of these components would cause the toxic effects. However, in the range of concentrations comparable to those used in our experiments $(94 \mu \mathrm{g} / \mathrm{mL}$ for sesame oil, $3 \mu \mathrm{g} / \mathrm{mL}$ for egg lecithin, and $5 \mu \mathrm{g} / \mathrm{mL}$ for Tween- 80 , according to a maximum concentration of $500 \mu \mathrm{g} / \mathrm{mL}$ of LNs and the percentage of raw materials added during fabrication), none of the major constituents showed any toxicity (Figure S2). Even with much higher concentrations $(1 \mathrm{mg} / \mathrm{mL})$, no influence was observed during 24-h-application of either egg lecithin or sesame oil. However, the findings were less clear after treatment with Tween- 80 , because it could cause cell loss at a concentration of $30 \mu \mathrm{g} / \mathrm{mL}$. This is a level that is only six times higher than the maximum concentration used in our experiments. Together with the fact that a maximum concentration of $0.05 \%$ of Tween-80 did not induce irritation in animals, ${ }^{16}$ we hypothesized that Tween- 80 can exert certain degree of cytotoxicity on isolated macrophages in relatively high concentrations, but in lower concentrations, it is generally safe. However, as a result of the deliverability of LNs in vitro and phagocytic activity of the macrophages, more Tween- 80 would enter into the cytosol of the cells and hence affect the integrity of mitochondria membranes, which could lead to apoptosis via a mitochondrial-dependent pathway. It would, therefore, be interesting to determine a detailed mechanism for this toxicity and also search for a good substitute for Tween- 80 that could be used for the fabrication of LNs.

\section{Conclusions}

In summary, we observed apoptotic effects of LNs on Kupffer cells in the liver and Raw264.7 cells in vitro. These effects occurred in a dose- and time-dependent manner via a mitochondrial-dependent apoptotic pathway. This ability to ablate Kupffer cells in mice makes these LNs a potential tool for elucidating the functions and kinetics of Kupffer cells in the physiologic and pathologic processes that can occur in the liver. Meanwhile, further studies are needed to determine whether LNs can induce apoptosis in other tissue-specific types of macrophages such as alveolar macrophages, microglia, sinus histiocytes, and intestinal macrophages.

\section{Availability of data and material}

The datasets used and/or analyzed during the current study are available from the corresponding author on reasonable request.

\section{Ethics approval and consent to participate}

The care and use of animals and experimental protocols for this study was carried out according to the Guide for Animal Experimentation, South-Central University for Nationalities and the Committee of Research Facilities for Laboratory Animal Sciences, South-Central University for Nationalities, China. The protocols were approved by the Committee on the Ethics of Animal Experiments of the South-Central University for Nationalities, China (permit number: 2018-SCUEC-AEC011).

\section{Research highlights}

- Lipid nanoparticles alone could induce apoptosis on Raw264.7 cells in a dose- and time-dependent manner.

- Acute or chronic application of lipid nanoparticles could lead to loss of murine hepatic Kupffer cells.

- This apoptotic effect works via a mitochondrial-dependent Bax/Bcl-2 pathway. 


\section{Acknowledgments}

The author would like to thank Xia-Lin Huang and QiuFang Qin for excellent technical support. This work was supported by the National Natural Science Foundation of China (31400705, 81673711, 81573887).

\section{Author contributions}

All authors contributed to data analysis, drafting or revising the article, gave final approval of the version to be published, and agree to be accountable for all aspects of the work.

\section{Disclosure}

The authors report no conflicts of interest in this work.

\section{References}

1. Danaei M, Dehghankhold M, Ataei S, et al. Impact of particle size and polydispersity index on the clinical applications of lipidic nanocarrier systems. Pharmaceutics. 2018;10:2. doi:10.3390/ pharmaceutics 10020057

2. Choi YH, Han HK. Nanomedicines: current status and future perspectives in aspect of drug delivery and pharmacokinetics. J Pharm Investig. 2018;48(1):43-60. doi:10.1007/s40005-017-0370-4

3. Palmer BC, DeLouise LA. Nanoparticle-enabled transdermal drug delivery systems for enhanced dose control and tissue targeting. Molecules. 2016;21:12. doi:10.3390/molecules21121719

4. Raemdonck K, Braeckmans K, Demeester J, De Smedt SC. Merging the best of both worlds: hybrid lipid-enveloped matrix nanocomposites in drug delivery. Chem Soc Rev. 2014;43(1):444-472. doi:10.1039/ c3cs60299k

5. Lim SB, Banerjee A, Onyuksel H. Improvement of drug safety by the use of lipid-based nanocarriers. J Control Release. 2012;163(1):34-45. doi:10.1016/j.jconrel.2012.06.002

6. Onoue S, Yamada S, Chan HK. Nanodrugs: pharmacokinetics and safety. Int J Nanomedicine. 2014;9:1025-1037. doi:10.2147/IJN. S38378

7. Mora-Huertas CE, Fessi H, Elaissari A. Polymer-based nanocapsules for drug delivery. Int J Pharm. 2010;385(1-2):113-142. doi:10.1016/j. ijpharm.2009.10.018

8. Yao M, Xiao H, McClements DJ. Delivery of lipophilic bioactives: assembly, disassembly, and reassembly of lipid nanoparticles. Annu Rev Food Sci Technol. 2014;5:53-81. doi:10.1146/annurev-food072913-100350

9. Lin Q, Chen J, Zhang Z, Zheng G. Lipid-based nanoparticles in the systemic delivery of siRNA. Nanomedicine (Lond). 2014;9(1):105120. doi:10.2217/nnm.13.192
10. Kauffman KJ, Dorkin JR, Yang JH, et al. Optimization of lipid nanoparticle formulations for mRNA delivery in vivo with fractional factorial and definitive screening designs. Nano Lett. 2015;15 (11):7300-7306. doi:10.1021/acs.nanolett.5b02497

11. Charoenputtakun P, Pamornpathomkul B, Opanasopit P, Rojanarata T, Ngawhirunpat T. Terpene composited lipid nanoparticles for enhanced dermal delivery of all-trans-retinoic acids. Biol Pharm Bull. 2014;37(7):1139-1148.

12. Weber S, Zimmer A, Pardeike J. Solid lipid nanoparticles (SLN) and nanostructured lipid carriers (NLC) for pulmonary application: a review of the state of the art. Eur J Pharm Biopharm. 2014;86 (1):7-22.

13. Gainza G, Pastor M, Aguirre JJ, et al. A novel strategy for the treatment of chronic wounds based on the topical administration of rhEGF-loaded lipid nanoparticles: in vitro bioactivity and in vivo effectiveness in healing-impaired $\mathrm{db} / \mathrm{db}$ mice. J Control Release. 2014;185:51-61. doi:10.1016/j.jconrel.2014.04.032

14. Zhang WK, Gu HW, Li XJ, et al. The dark side of "the force" - lipid nanoparticles enhance the oncogenesis of diethylnitrosamine and result in liver cancer in mice. Nanomedicine. 2017;13(2):701-711. doi:10.1016/j.nano.2016.09.017

15. Seki E, Tsutsui H, Iimuro Y, et al. Contribution of Toll-like receptor/ myeloid differentiation factor 88 signaling to murine liver regeneration. Hepatology. 2005;41(3):443-450. doi:10.1002/hep.20603

16. Thomson AW, Knolle PA. Antigen-presenting cell function in the tolerogenic liver environment. Nat Rev Immunol. 2010;10(11):753766. doi: $10.1038 /$ nri2858

17. Tsutsui H, Nishiguchi S. Importance of Kupffer cells in the development of acute liver injuries in mice. Int J Mol Sci. 2014;15(5):77117730. doi:10.3390/ijms15057711

18. Ju C, Reilly TP, Bourdi M, et al. Protective role of Kupffer cells in acetaminophen-induced hepatic injury in mice. Chem Res Toxicol. 2002;15(12):1504-1513.

19. Ramachandran P, Iredale JP. Macrophages: central regulators of hepatic fibrogenesis and fibrosis resolution. J Hepatol. 2012;56 (6):1417-1419. doi:10.1016/j.jhep.2011.10.026

20. Chiang DJ, Pritchard MT, Nagy LE. Obesity, diabetes mellitus, and liver fibrosis. Am J Physiol Gastrointest Liver Physiol. 2011;300(5): G697-G702. doi:10.1152/ajpgi.00426.2010

21. Zhao X, Qi C, Li Y, Tang H, Wei L, Yang X. Doxorubicin and curcumin co-delivery by lipid nanoparticles for enhanced treatment of diethylnitrosamine-induced hepatocellular carcinoma in mice. Eur J Pharm Biopharm. 2015;93:27-36. doi:10.1016/j.ejpb.2015.03.003

22. Xiaojing Zhao QC, Liu W, Li Y, Tang H, Liu X, Yang X. Codelivery of doxorubicin and curcumin with lipid nanoparticles results in improved efficacy of chemotherapy in liver cancer. Int $J$ Nanomedicine. 2015;10(default):257-270. doi:10.2147/IJN.S73322

23. Mosser DM, Edwards JP. Exploring the full spectrum of macrophage activation. Nat Rev Immunol. 2008;8(12):958-969. doi:10.1038/ nri2448

24. Dixon LJ, Barnes M, Tang H, Pritchard MT, Nagy LE. Kupffer cells in the liver. Compr Physiol. 2013;3(2):785-797. doi:10.1002/cphy. c120026 


\section{Supplementary material}
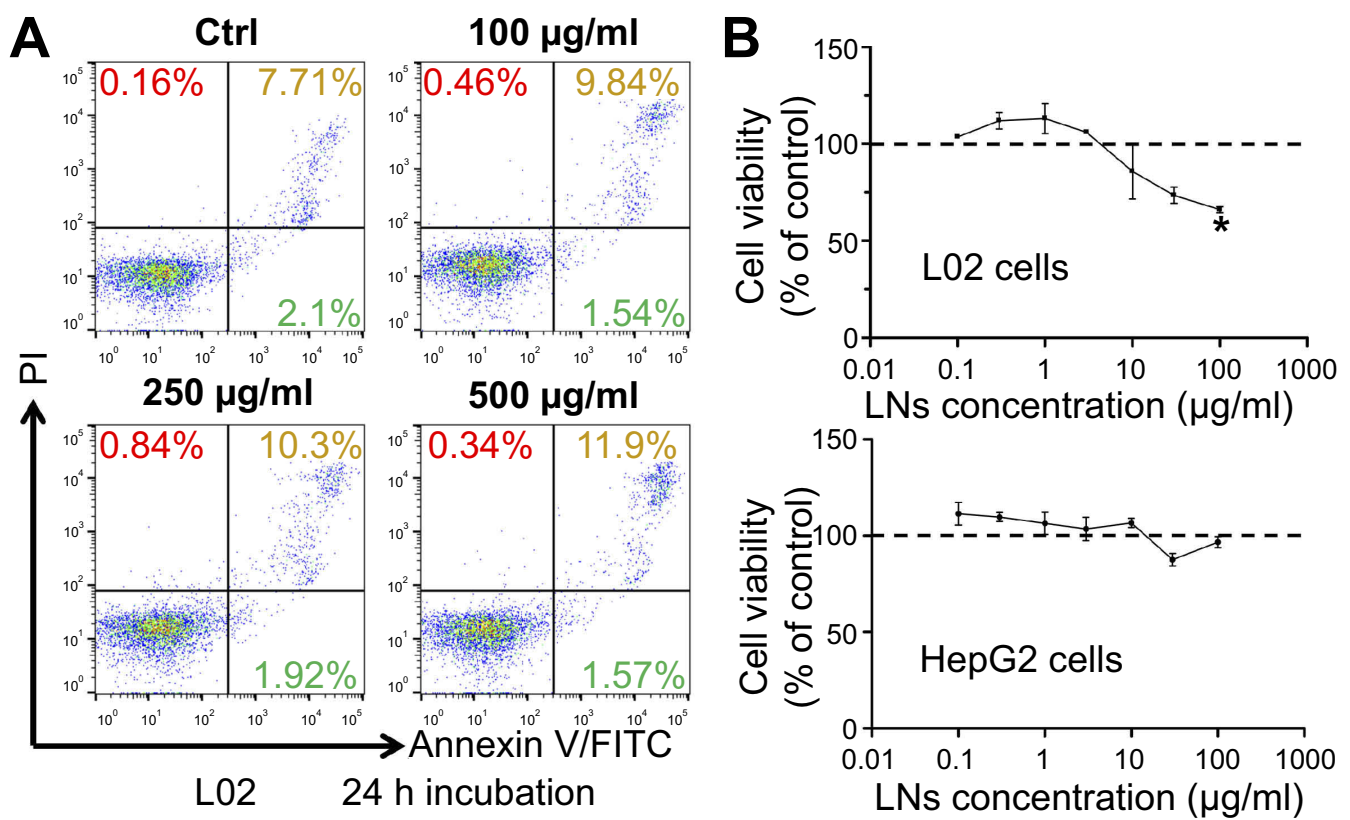

Figure SI LNs did not cause obvious damage on hepatocytes. (A) Apoptotic detection of L02 cells treated with various concentrations of LNs. (B) MTT analysis of L02 and HepG-2 cells treated with LNs.

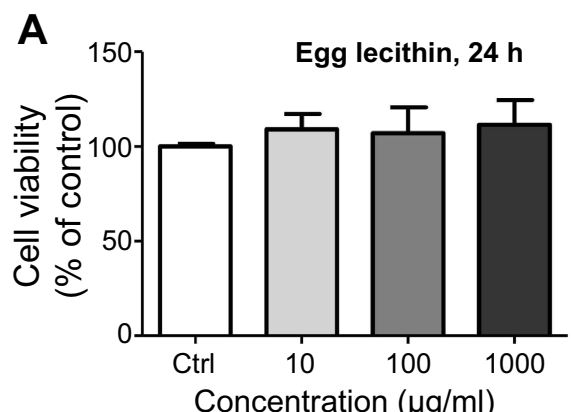

C

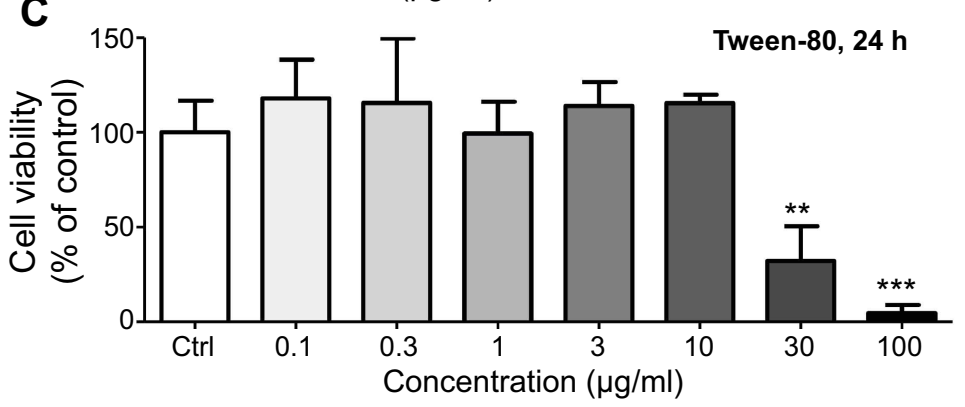

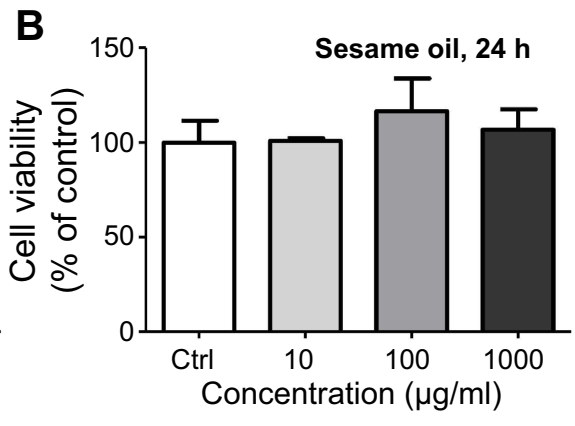

Tween-80, $24 \mathrm{~h}$

Figure S2 MTT analysis of major components in LNs. (A) Cell viability of different concentrations of egg yolk lecithin on Raw264.7 cells. (B) Cell viability of different concentrations of sesame oil on Raw264.7 cells. (C) Cell viability of different concentrations of Tween-80 on Raw264.7 cells. ** and ***represent significant difference from control with $P<0.0 \mathrm{I}$ and $0.00 \mathrm{I}$, respectively. 
Table SI No obvious change was detected in serum biochemistry study among groups after short-term application of LNs

\begin{tabular}{|l|l|l|l|l|l|l|}
\hline & ALT (U/L) & AST (U/L) & ALP (U/L) & TBIL (mg/dL) & TG (mg/dL) & TCHO (mg/dL) \\
\hline Control & $34.00 \pm 4.78$ & $104.33 \pm 9.71$ & $197.67 \pm 26.03$ & $0.28 \pm 0.06$ & $232.17 \pm 40.98$ & $116.50 \pm 8.40$ \\
$10 \mathbf{~ m g / k g}$ & $39.17 \pm 5.37$ & $104.33 \pm 9.71$ & $217.67 \pm 15.66$ & $0.28 \pm 0.05$ & $194.67 \pm 14.67$ & $131.33 \pm 5.30$ \\
$\mathbf{3 0} \mathbf{~ m g / k g}$ & $33.00 \pm 1.37$ & $115.50 \pm 14.97$ & $201.17 \pm 25.87$ & $0.28 \pm 0.03$ & $242.17 \pm 40.21$ & $115.00 \pm 6.00$ \\
\hline
\end{tabular}

Abbreviation: ALT, alanine transaminase; AST, aspartate transaminase; ALP, alkaline phosphatase; TBIL, total bilirubin; TG, triglyceride; TCHO, total cholesterol; ALT, AST, ALP and TBIL partly reflected the liver function while TG and TCHO revealed lipid metabolism.

\section{Publish your work in this journal}

The International Journal of Nanomedicine is an international, peerreviewed journal focusing on the application of nanotechnology in diagnostics, therapeutics, and drug delivery systems throughout the biomedical field. This journal is indexed on PubMed Central, MedLine, CAS, SciSearch $\AA$, Current Contents $₫ /$ Clinical Medicine,
Journal Citation Reports/Science Edition, EMBase, Scopus and the Elsevier Bibliographic databases. The manuscript management system is completely online and includes a very quick and fair peer-review system, which is all easy to use. Visit http://www.dovepress.com/ testimonials.php to read real quotes from published authors. 